


\title{
Relative Chern characters for nilpotent ideals
}

\author{
G. Cortiñas ${ }^{1}$ and C. Weibel ${ }^{2}$ \\ 1 Dep. Matemática, Ciudad Universitaria Pab 1, C1428EGA Buenos Aires, Argentina. gcorti@dm.uba.ar \\ 2 Dept. of Mathematics, Rutgers University, New Brunswick, NJ 08901, USA. weibel@math.rutgers.edu
}

\section{Introduction}

When $A$ is a unital ring, the absolute Chern character is a group homomorphism $c h_{*}: K_{*}(A) \rightarrow$ $H N_{*}(A)$, going from algebraic $K$-theory to negative cyclic homology (see $[7,11.4]$ ). There is also a relative version, defined for any ideal $I$ of $A$ :

$$
c h_{*}: K_{*}(A, I) \rightarrow H N_{*}(A, I) .
$$

Now suppose that $A$ is a $\mathbb{Q}$-algebra and that $I$ is nilpotent. In this case, Goodwillie proved in [4] that (1.1) is an isomorphism. His proof uses another character

$$
c h_{*}^{\prime}: K_{*}(A, I) \rightarrow H N_{*}(A, I)
$$

which is defined only when $I$ is nilpotent and $\mathbb{Q} \subset A$. Goodwillie showed that (1.2) is an isomorphism whenever it is defined, and that it coincides with (1.1) when $I^{2}=0$. Using this and a 5 -Lemma argument, he deduced that (1.1) is an isomorphism for any nilpotent $I$. The question of whether $c h_{*}$ and $c h_{*}^{\prime}$ agree for general nilpotent ideals $I$ was left open in [4], and announced without proof in $[7,11.4 .11]$. This paper answers the question, proving in Theorem 6.5.1 that

$$
c h_{*}=c h_{*}^{\prime} \text { for all nilpotent ideals } I .
$$

Here are some applications of (1.3). Let $I$ a nilpotent ideal in a commutative $\mathbb{Q}$-algebra $A$. Cathelineau proved in [1] that (1.2) preserves the direct sum decomposition coming from the eigenspaces of $\lambda$-operations and/or Adams operations. By (1.3), the relative Chern character (1.1) preserves the direct sum decomposition for nilpotent ideals. Next, Cathelineau's result and (1.3) are used in [2] to prove that the absolute Chern character $c h_{*}$ also preserves the direct sum decomposition. In addition, our result (1.3) can be used to strengthen Ginot's results in [3].

This paper is laid out according to the following plan. In section 2 we show that the bar complex $\mathrm{B}(\mathrm{H})$ of a cocommutative Hopf algebra $\mathrm{H}$ has a natural cyclic module structure. The case $\mathrm{H}=k[G]$ is well known, and the case of enveloping algebras is implicit in [6]. In section 3, we relate this construction to the usual cyclic module of the algebra underlying $\mathrm{H}$. In section 4 we consider a Lie algebra $\mathfrak{g}$ and factor the Loday-Quillen map $\wedge^{n} \mathfrak{g} \rightarrow C_{n-1}^{\lambda}(U \mathfrak{g})$ through our construction. In section 5 we consider a nilpotent Lie algebra $\mathfrak{g}$ and its associated nilpotent group $G$ and relate the constructions for $U \mathfrak{g}$ and $\mathbb{Q}[G]$ using the Mal'cev theory of [10, App. A]. In section 6 , we review the definitions of $c h_{*}$ and $c h_{*}^{\prime}$ and prove our main theorem, that (1.3) holds.

\footnotetext{
* Cortiñas is supported by FCEyN-UBA and CONICET, and partially supported by grants ANPCyT PICT 2006-0836, UBACyT-X057, and MEC MTM00958.

Weibel's research was supported by NSA grant MSPF-04G-184 and NSF grant DMS-0801060
} 


\section{Notation}

If $M=\left(M_{*}, b, B\right)$ is a mixed complex $([7,2.5 .13])$, we will write $H H(M)$ for the chain complex $\left(M_{*}, b\right)$, and $H N(M)$ for the total complex of Connes' left half-plane $(b, B)$-complex (written as $\mathcal{B} M^{-}$in $\left.[7,5.1 .7]\right)$. By definition, the homology of $H H(M)$ is the Hochschild homology $H H_{*}(M)$ of $M$, the homology of $H N(M)$ is the negative cyclic homology $H N_{*}(M)$ of $M$, and the projection $\pi: H N(M) \rightarrow H H(M)$ induces the canonical map $H N_{*}(M) \rightarrow H H_{*}(M)$.

We refer the reader to $[7,2.5 .1]$ for the notion of a cyclic module. There is a canonical cyclic $k$-module $C(A)$ associated to any algebra $A$, with $C_{n}(A)=A^{\otimes n+1}$, whose underlying simplicial module $(C, b)$ is the Hochschild complex (see [7, 2.5.4]). We will write $H H(A)$ and $H N(A)$ in place of the more awkward expressions $H H(C(A))$ and $H N(C(A))$.

We write $C_{\geq n}$ for the good truncation $\tau_{\geq n} C$ of a chain complex $C$ [13, 1.2.7]. If $n \geq 0$, then $C_{\geq n}$ can and will be regarded as a simplicial module via the Dold-Kan correspondence $[13,8.4]$.

\section{Cyclic homology of cocommutative Hopf algebras}

If $A$ is the group algebra of a group $G$, then the bar resolution $\mathrm{E}(A)=k[\mathrm{E} G]$ admits a cyclic $G$ module structure and the bar complex $\mathrm{B}(A)=k[\mathrm{~B} G]$ also admits a cyclic $k$-module structure [7]. In this section, we show that the cyclic modules $\mathrm{E}(A)$ and $\mathrm{B}(A)$ can be defined for any cocommutative Hopf algebra $A$.

\subsection{Bar resolution and bar complex of an augmented algebra}

Let $k$ be a commutative ring, and $A$ an augmented unital $k$-algebra, with augmentation $\epsilon: A \rightarrow k$. We write $\mathrm{E}(A)$ for the bar resolution of $k$ as a left $A$-module $([13,8.6 .12])$; this is the simplicial $A$-module $\mathrm{E}_{n}(A)=A^{\otimes n+1}$, whose face and degeneracy operators are given by

$$
\begin{gathered}
\mu_{i}: \mathrm{E}_{n}(A) \rightarrow \mathrm{E}_{n-1}(A) \quad(i=0, \ldots, n) \\
\mu_{i}\left(a_{0} \otimes \cdots \otimes a_{n}\right)=a_{0} \otimes \cdots \otimes a_{i} a_{i+1} \otimes \cdots \otimes a_{n} \quad(i<n) \\
\mu_{n}\left(a_{0} \otimes \cdots \otimes a_{n}\right)=\varepsilon\left(a_{n}\right) a_{0} \otimes \cdots \otimes a_{n-1} \\
s_{j}: \mathrm{E}_{n}(A) \rightarrow \mathrm{E}_{n+1}(A) \quad(j=0, \ldots, n) \\
s_{j}\left(a_{0} \otimes \cdots \otimes a_{n}\right)=a_{0} \otimes \cdots \otimes a_{j} \otimes 1 \otimes a_{j+1} \otimes \cdots \otimes a_{n}
\end{gathered}
$$

We write $\partial^{\prime}$ for the usual boundary map $\sum_{i=0}^{n}(-1)^{i} \mu_{i}: \mathrm{E}_{n}(A) \rightarrow \mathrm{E}_{n-1}(A)$. The augmentation induces a quasi-isomorphism $\epsilon: \mathrm{E}(A) \rightarrow k$. The unit $\eta: k \rightarrow A$ is a $k$-linear homotopy inverse of $\epsilon$; we have $\epsilon \eta=1$ and the extra degeneracy $s: \mathrm{E}(A)_{n} \rightarrow \mathrm{E}(A)_{n+1}$,

$$
s(x)=1 \otimes x
$$

satisfies $1-\eta \epsilon=\left[\partial^{\prime}, s\right]$. The bar complex of $A$ is $\mathrm{B}(A)=k \otimes_{A} \mathrm{E}(A) ; \mathrm{B}_{n}(A)=A^{\otimes n}$. We write $\partial=1 \otimes \partial^{\prime}: \mathrm{B}(A)_{n} \rightarrow \mathrm{B}(A)_{n-1}$ for the induced boundary map, and $\mathrm{E}(A)_{\text {norm }}$ and $\mathrm{B}(A)_{\text {norm }}$ for the normalized complexes.

\subsection{The cyclic module of a cocommutative coalgebra}

If $C$ is a $k$-coalgebra, with counit $\epsilon: C \rightarrow k$ and coproduct $\Delta$, we have a simplicial $k$-module $R(C)$, with $R_{n}(C)=C^{\otimes n+1}$, and face and degeneracy operators given by

$$
\begin{gathered}
\varepsilon_{i}: R_{n}(C) \rightarrow R_{n-1}(C) \quad(i=0, \ldots, n) \\
\varepsilon_{i}\left(c_{0} \otimes \cdots \otimes c_{n}\right)=\epsilon\left(c_{i}\right) c_{0} \otimes \cdots \otimes c_{i-1} \otimes c_{i+1} \otimes \ldots c_{n} \\
\Delta_{i}: R_{n}(C) \rightarrow R_{n+1}(C) \quad(i=0, \ldots, n) \\
\Delta_{i}\left(c_{0} \otimes \cdots \otimes c_{n}\right)=c_{0} \otimes \ldots c_{i-1} \otimes c_{i}^{(0)} \otimes c_{i}^{(1)} \otimes \cdots \otimes c_{n}
\end{gathered}
$$


Here and elsewhere we use the (summationless) Sweedler notation $\Delta(c)=c^{(0)} \otimes c^{(1)}$ of [11]. We remark that each $R_{n}(C)$ has a coalgebra structure, and that the face maps $\varepsilon_{i}$ are coalgebra homomorphisms. If in addition $C$ is cocommutative, then the degeneracies $\Delta_{j}$ are also coalgebra homomorphisms, and $R(C)$ is a simplicial coalgebra. In fact $R_{n}(C)$ is the product of $n+1$ copies of $C$ in the category of cocommutative coalgebras, and $R(C)$ is a particular case of the usual product simplicial resolution of an object in a category with finite products, which is a functor not only on the simplicial category of finite ordinals and monotone maps, but also on the larger category $\mathfrak{F i n}$ with the same objects, but where a homomorphism is just any set theoretic map, not necessarily order preserving. By $[7,6.4 .5]$ we have:

Lemma 2.2.1. For a cocommutative coalgebra $C$, the simplicial module $R(C)$ has the structure of a cyclic $k$-module, with cyclic operator

$$
\lambda\left(c_{0} \otimes \cdots \otimes c_{n}\right)=(-1)^{n} c_{n} \otimes c_{0} \otimes \cdots \otimes c_{n-1} .
$$

Remark 2.2.2. Let $G$ be a group; write $k[G]$ for the group algebra. Note $k[G]$ is a Hopf algebra, and in particular, a coalgebra, with coproduct determined by $\Delta(g)=g \otimes g$. The cyclic module $R(k[G])$ thus defined is precisely the cyclic module whose associated cyclic bicomplex was considered by Karoubi in $[5,2.21]$, where it is written $\tilde{C}_{* *}(G)$.

\subsection{The case of Hopf algebras}

Let $\mathrm{H}$ be a Hopf algebra with unit $\eta$, counit $\epsilon$ and antipode $S$. We shall assume that $S^{2}=1$, which is the case for all cocommutative Hopf algebras. We may view $R(\mathrm{H})$ as a simplicial left $\mathrm{H}$-module via the diagonal action:

$$
a \cdot\left(h_{0} \otimes \cdots \otimes h_{n}\right)=a^{(0)} h_{0} \otimes \cdots \otimes a^{(n)} h_{n} .
$$

Consider the maps (defined using summationless Sweedler notation):

$$
\begin{gathered}
\alpha: \mathrm{E}_{n}(\mathrm{H}) \rightarrow R_{n}(\mathrm{H}), \\
\alpha\left(h_{0} \otimes \cdots \otimes h_{n}\right)=h_{0}^{(0)} \otimes h_{0}^{(1)} h_{1}^{(0)} \otimes \cdots \otimes h_{0}^{(n)} h_{1}^{(n-1)} \cdots h_{n-1}^{(1)} h_{n} \\
\beta: R_{n}(\mathrm{H}) \rightarrow \mathrm{E}_{n}(\mathrm{H}), \\
\beta\left(h_{0} \otimes \cdots \otimes h_{n}\right)=h_{0}^{(0)} \otimes\left(S h_{0}^{(1)}\right) h_{1}^{(0)} \otimes \cdots \otimes\left(S h_{n-1}^{(1)}\right) h_{n}
\end{gathered}
$$

A straightforward computation reveals:

Lemma 2.3.3. The maps (2.3.1) and (2.3.2) are inverse isomorphisms of simplicial $\mathrm{H}$-modules: $\mathrm{E}_{n}(\mathrm{H}) \cong R_{n}(\mathrm{H})$.

\subsection{Cyclic complexes of cocommutative Hopf algebras}

From now on, we shall assume that $\mathrm{H}$ is a cocommutative Hopf algebra. In this case the cyclic operator $\lambda: R(\mathrm{H}) \rightarrow R(\mathrm{H})$ of 2.2.1 is a homomorphism of $\mathrm{H}$-modules. Thus $R(\mathrm{H})$ is a cyclic $\mathrm{H}$ module, and we can use the isomorphisms $\alpha$ and $\beta$ of Lemma 2.3.3 to translate this structure to the bar resolution $\mathrm{E}(\mathrm{H})$. We record this as a corollary:

Corollary 2.4.1. When $\mathrm{H}$ is a cocommutative Hopf algebra, $\mathrm{E}(\mathrm{H})$ is a cyclic $\mathrm{H}$-module, and $\mathrm{B}(\mathrm{H})=k \otimes_{\mathrm{H}} \mathrm{E}(\mathrm{H})$ is a cyclic $k$-module. The cyclic operator $t:=\beta \lambda \alpha$ on $\mathrm{E}(\mathrm{H})$ is given by the formulas $t(h)=h, t\left(h_{0} \otimes h_{1}\right)=-h_{0} h_{1}^{(0)} \otimes S h_{1}^{(1)}$ and:

$$
t\left(h_{0} \otimes \cdots \otimes h_{n}\right)=(-1)^{n} h_{0} h_{1}^{(0)} \ldots h_{n}^{(0)} \otimes S\left(h_{1}^{(1)} \ldots h_{n}^{(1)}\right) \otimes h_{1}^{(2)} \otimes \cdots \otimes h_{n-1}^{(2)} .
$$


Remark 2.4.2. If $g_{0}, \ldots, g_{n} \in \mathrm{H}$ are grouplike elements then

$$
t\left(g_{0} \otimes \cdots \otimes g_{n}\right)=(-1)^{n} g_{0} \ldots g_{n} \otimes\left(g_{1} \ldots g_{n}\right)^{-1} \otimes g_{1} \otimes \cdots \otimes g_{n-1} .
$$

In particular, for $\mathrm{H}=k[G], \mathrm{B}(k[G])$ with the cyclic structure of 2.4 .1 is the cyclic module associated to the cyclic set $\mathrm{B}(G, 1)$ of $[7,7.3 .3]$.

For the extra degeneracy of $[7,2.5 .7]$,

$$
s^{\prime}=(-1)^{n+1} t s_{n}: \mathrm{E}_{n}(\mathrm{H}) \rightarrow \mathrm{E}_{n+1}(\mathrm{H}),
$$

it is immediate from (2.1.1) and 2.4.1 that $s^{\prime}$ is signfree:

$$
s^{\prime}\left(h_{0} \otimes \cdots \otimes h_{n}\right)=h_{0} h_{1}^{(0)} \ldots h_{n}^{(0)} \otimes S\left(h_{1}^{(1)} \ldots h_{n}^{(1)}\right) \otimes h_{1}^{(2)} \otimes \cdots \otimes h_{n}^{(2)} .
$$

By 2.4.1, the Connes' operator $B^{\prime}$ is the $\mathrm{H}$-module homomorphism:

$$
B^{\prime}=(1-t) s^{\prime} \sum_{i=0}^{n} t^{i}: \mathrm{E}_{n}(\mathrm{H}) \rightarrow \mathrm{E}_{n+1}(\mathrm{H}) .
$$

(See [8, p. 569].) We write $B: \mathrm{B}(\mathrm{H})_{n} \rightarrow \mathrm{B}(\mathrm{H})_{n+1}$ for the induced $k$-module map.

Definition 2.4.5. We define the mixed $\mathrm{H}$-module complex $\mathrm{M}^{\prime}=\mathrm{M}^{\prime}(\mathrm{H})$, and the mixed $k$-module complex $\mathrm{M}=\mathrm{M}(\mathrm{H})$ to be the normalized mixed complexes associated to the cyclic modules $\mathrm{E}_{*}(\mathrm{H})$ and $\mathrm{B}_{*}(\mathrm{H})$ of 2.4.1:

$$
\begin{gathered}
\mathrm{M}^{\prime}(\mathrm{H}):=\left(\mathrm{E}_{*}(\mathrm{H})_{\text {norm }}, \partial^{\prime}, B^{\prime}\right) \\
\mathrm{M}(\mathrm{H}):=k \otimes_{\mathrm{H}} \mathrm{M}^{\prime}(\mathrm{H})=\left(\mathrm{B}_{*}(\mathrm{H})_{\text {norm }}, \partial, B\right) .
\end{gathered}
$$

Remark 2.4.6. Consider the map $s^{\prime \prime}=(-1)^{n} s_{n}: \mathrm{E}_{n}(\mathrm{H}) \rightarrow \mathrm{E}_{n+1}(\mathrm{H})$, and set $B^{\prime \prime}=-t s^{\prime \prime} N$, where as usual $N=\sum t^{i}$ is the norm map. Then $B^{\prime}=B^{\prime \prime}$ on $\mathrm{E}(\mathrm{H})_{\text {norm }}$ because the relations $s_{0} t_{n}=$ $(-1)^{n} t_{n+1}^{2} s_{n}$ and $t N=N$ yield for all $x \in \mathrm{E}_{n}(\mathrm{H})$ :

$$
\begin{aligned}
\left(B^{\prime \prime}-B^{\prime}\right)(x) & =\left((-1)^{n+1} t s_{n} N+(-1)^{n}(1-t) t s_{n} N\right)(x) \\
& =(-1)^{n}\left(-t s_{n} N(x)+t s_{n} N(x)-t^{2} s_{n} N(x)\right) \\
& =-s_{0} t N(x)=-s_{0} N(x) \equiv 0 \quad \text { in } \mathrm{E}(\mathrm{H})_{\text {norm }} .
\end{aligned}
$$

Lemma 2.4.7. The map $B^{\prime}: \mathrm{E}(\mathrm{H})_{\text {norm }} \rightarrow \mathrm{E}(\mathrm{H})_{\text {norm }}[1]$ induced by (2.4.4) is given by the explicit formula:

$$
\begin{gathered}
B^{\prime}\left(h_{0} \otimes \cdots \otimes h_{n}\right)= \\
\sum_{i=0}^{n}(-1)^{n i} h_{0} h_{1}^{(0)} \cdots h_{n-i}^{(0)} \otimes h_{n-i+1}^{(0)} \otimes \cdots \otimes h_{n}^{(0)} \otimes S\left(h_{1}^{(1)} \cdots h_{n}^{(1)}\right) \otimes h_{1}^{(2)} \otimes \cdots \otimes h_{n-i}^{(2)} .
\end{gathered}
$$

Proof. For convenience, let us write $S h^{(1)}$ for $S\left(h_{1}^{(1)} \cdots h_{n}^{(1)}\right)$. It follows from 2.4.1, cocomutativity and induction on $i$ that $t^{i}\left(h_{0}\right)=h_{0}$, and if $i \leq n$ then:

$$
\begin{gathered}
t^{i}\left(h_{0} \otimes \cdots \otimes h_{n}\right)= \\
(-1)^{n i} h_{0} h_{1}^{(0)} \ldots h_{n-i+1}^{(0)} \otimes h_{n-i+2}^{(0)} \otimes \cdots \otimes h_{n}^{(0)} \otimes S h^{(1)} \otimes h_{1}^{(2)} \otimes \cdots \otimes h_{n-i}^{(2)} .
\end{gathered}
$$

Let $s^{\prime \prime}=(-1)^{n} s_{n}$ be as in Remark 2.4.6 and set $m=n(i+1)+1$. We have

$$
\begin{gathered}
-t s^{\prime \prime} t^{i}\left(h_{0} \otimes \cdots \otimes h_{n}\right)=(-1)^{n+1} t s_{n} t^{i}\left(h_{0} \otimes \cdots \otimes h_{n}\right) \\
=(-1)^{m} t\left(h_{0} h_{1}^{0} \ldots h_{n-i+1}^{(0)} \otimes h_{n-i+2}^{(0)} \otimes \cdots \otimes h_{n}^{(0)} \otimes S h^{(1)} \otimes h_{1}^{(2)} \otimes \cdots \otimes h_{n-i}^{(2)} \otimes 1\right) \\
=(-1)^{n i} h_{0} h_{1}^{(0)} \ldots h_{n-i}^{(0)} \otimes h_{n-i+1}^{(0)} \otimes \cdots \otimes h_{n}^{(0)} \otimes S h^{(1)} \otimes h_{1}^{(2)} \otimes \cdots \otimes h_{n-i}^{(2)} .
\end{gathered}
$$

Now sum up over $i$ to get $B^{\prime \prime}$ and use Remark 2.4.6. 
Corollary 2.4.8. Suppose that $x_{1}, \ldots, x_{n}$ are primitive elements of $\mathrm{H}$, and $h \in \mathrm{H}$. Then $B^{\prime}(h \otimes$ $\left.x_{1} \otimes \cdots \otimes x_{n}\right)=0$ in $\mathrm{E}(\mathrm{H})_{\text {norm }}$.

Proof. When $x$ is primitive, $x^{(0)} \otimes x^{(1)} \otimes x^{(2)}$ is $1 \otimes 1 \otimes x+2 \otimes x \otimes 1+x \otimes 1 \otimes 1$. By Lemma 2.4.7, $B^{\prime}\left(h \otimes x_{1} \otimes \cdots \otimes x_{n}\right)$ is a sum of terms of the form

$$
\pm h^{\prime} \otimes x_{j+1}^{(0)} \otimes \cdots \otimes x_{n}^{(0)} \otimes S\left(x_{1}^{(1)} \cdots x_{n}^{(1)}\right) \otimes x_{1}^{(2)} \otimes \cdots \otimes x_{j}^{(2)} .
$$

By inspection, each such term is degenerate, and vanishes in $\mathrm{E}(\mathrm{H})_{\text {norm }}$.

\subsection{Adic filtrations and completion}

As usual, we can use an adic topology on a Hopf algebra to define complete Hopf algebras, and topological versions of the above complexes.

First we recall some generalities about filtrations and completions of $k$-modules, following [10]. There is a category of filtered $k$-modules and filtration-preserving maps; a filtered module $V$ is a module equipped with a decreasing filtration $V=\mathcal{F}_{0}(V) \supseteq \mathcal{F}_{1}(V) \supseteq \cdots$. The completion of $V$ is $\hat{V}=\lim _{n} V / \mathcal{F}_{n} V$; it is a filtered module in the evident way. If $W$ is another filtered $k$-module, then the tensor product $V \otimes W$ is a filtered module with filtration

$$
\mathcal{F}_{n}(V \otimes W)=\sum_{p+q=n} \operatorname{image}\left(\mathcal{F}_{p} V \otimes \mathcal{F}_{q} W \rightarrow V \otimes W\right) .
$$

We define $\hat{V} \hat{\otimes} \hat{W}$ to be $\widehat{\hat{V} \otimes \hat{W}}$. Note that

$$
\mathcal{F}_{n}(V \otimes W) \supseteq \operatorname{image}\left(\mathcal{F}_{n} V \otimes W+V \otimes \mathcal{F}_{n} W \rightarrow V \otimes W\right) \supseteq \mathcal{F}_{2 n}(V \otimes W) .
$$

Hence the topology defined by $\left\{\mathcal{F}_{n}(V \otimes W)\right\}$ is the same as that defined by $\{\operatorname{ker}(V \otimes W \rightarrow$ $\left.\left.V / \mathcal{F}_{n} V \otimes W / \mathcal{F}_{n} W\right)\right\}$. It follows that $\hat{V} \hat{\otimes} \hat{W}$ satisfies

$$
\hat{V} \hat{\otimes} \hat{W}=\widehat{V \otimes W} .
$$

All this has an obvious extension to tensor products of finitely many factors.

If $A$ is a filtered algebra (an algebra which is filtered as a $k$-module by ideals), then $\hat{A}$ is an algebra. If $I$ is an ideal then $\left\{I^{n}\right\}$ is called the $I$-adic filtration on $A$.

Now suppose that $\mathrm{H}$ is a cocommutative Hopf algebra, equipped with the $I$-adic filtration, where $I$ is both a (2-sided) ideal and a (2-sided) coideal of $\mathrm{H}$, closed under the antipode $S$ and satisfying $\epsilon(I)=0$. The coideal condition on $I$ means that

$$
\Delta(I) \subset \mathrm{H} \otimes I+I \otimes \mathrm{H} .
$$

This implies that $\Delta: \mathrm{H} \rightarrow \mathrm{H} \otimes \mathrm{H}$ is filtration-preserving; by (2.5.3) it induces a map $\hat{\Delta}: \hat{\mathrm{H}} \rightarrow \hat{\mathrm{H}} \hat{\otimes} \hat{\mathrm{H}}$, making $\hat{\mathrm{H}}$ into a complete Hopf algebra in the sense of [10].

Consider the induced filtrations (2.5.1) in $\mathrm{E}_{n}(\mathrm{H})$ and $\mathrm{B}_{n}(\mathrm{H})(n \geq 0)$. It is clear, from the formulas (2.1.1) and our assumption that $\epsilon(I)=0$, that the simplicial structures of $\mathrm{E}(\mathrm{H})$ and $\mathrm{B}(\mathrm{H})$ are compatible with the filtration; i.e., $\mathrm{E}(\mathrm{H})$ and $\mathrm{B}(\mathrm{H})$ are simplicial filtered objects. Since $I$ is a coideal closed under $S$, the formula in 2.4.1 implies that $t$ preserves the filtration. Thus $\mathrm{E}(\mathrm{H})$ and $\mathrm{B}(\mathrm{H})$ are cyclic filtered modules. It follows that $\mathrm{M}^{\prime}(\mathrm{H})$ and $\mathrm{M}(\mathrm{H})$ are filtered mixed complexes.

The identities (2.5.3) show that the completed objects $\hat{E}(H), \hat{B}(H)$, etc. depend only on the topological Hopf algebra $\hat{H}$, and can be regarded as its topological bar resolution, bar complex etc. which are defined similarly to their algebraic counterparts, but substituting $\hat{\otimes}$ for $\otimes$ everywhere. In this spirit, we shall write $E^{\text {top }}(\hat{H}), B^{\text {top }}(\hat{H})$, etc., for $\hat{E}(H), \hat{B}(H)$, etc. 


\section{Comparison with the cyclic module of the algebra $\mathbf{H}$}

Let $\mathrm{H}$ be a cocommutative Hopf algebra. In this section, we construct an injective cyclic module map $\tau: \mathrm{B}(\mathrm{H}) \rightarrow C(\mathrm{H})$, from the cyclic bar complex $\mathrm{B}(\mathrm{H})$ of 2.4 .1 to the canonical cyclic $k$-module $C(\mathrm{H})$ of the algebra underlying $\mathrm{H}([7,2.5 .4])$, and a lift $c$ of $\tau$ to the negative cyclic complex $H N(\mathrm{H})$ of $\mathrm{H}$.

In the group algebra case, these constructions are well-understood. The cyclic module inclusion $\tau: \mathrm{B}(k[G]) \subset C(k[G])$ is given in [7, 7.4]; see Example 3.2.2 below. Goodwillie proved in [4] that $\tau$ admits a natural lifting to a chain map $c: \mathrm{B}(k[G]) \rightarrow H N(k[G])$ to the negative cyclic complex, and that $c$ is unique up to natural homotopy. An explicit formula for such a lifting was given by Ginot [3], in the normalized, mixed complex setting.

\subsection{A natural section of the projection $\mathrm{HN}\left(\mathrm{M}^{\prime}(\mathrm{H})\right) \rightarrow H H\left(\mathrm{M}^{\prime}(\mathrm{H})\right)$.}

Recall from Definition 2.4.5 that $\mathrm{M}^{\prime}=\mathrm{M}^{\prime}(\mathrm{H})$ is a mixed complex whose underlying chain complex is $H H\left(\mathrm{M}^{\prime}\right)=\left(\mathrm{E}(\mathrm{H})_{\text {norm }}, \partial^{\prime}\right)$, and write $\pi^{\prime}$ for the projection from the negative cyclic complex $H N\left(\mathrm{M}^{\prime}\right)$ to $H H\left(\mathrm{M}^{\prime}\right)$. Following the method of Ginot [3], we shall define a natural $\mathrm{H}$-linear chain homomorphism $\Upsilon^{\prime}: H H\left(\mathrm{M}^{\prime}\right) \rightarrow H N\left(\mathrm{M}^{\prime}\right)$ such that $\pi^{\prime} \Upsilon^{\prime}=1$.

We shall use a technical lemma about maps between chain complexes of modules over a $k$-algebra A. Assume given all of the following:

(i) A homomorphism of chain $A$-modules $f: C \rightarrow D$, with $C_{n}=0$ for $n<n_{0}$.

(ii) A decomposition $C_{n} \cong A \otimes V_{n}$ for each $n$, where $V_{n}$ is a $k$-module.

(iii) A $k$-linear chain contraction $s$ for $D$.

Lemma 3.1.1. Given (i)-(iii), there is an A-linear chain contraction $\kappa^{f}$ of $f$, defined by $\kappa_{n_{0}}^{f}(a v)=$ asf $(v)$ and the inductive formula:

$$
\kappa_{n}^{f}: C_{n}=A \otimes V_{n} \rightarrow D_{n+1}, \quad \kappa_{n}^{f}(a v)=a s\left(f-\kappa_{n-1}^{f} d\right)(v) .
$$

Proof. We have to verify the formula $f(a v)=\kappa^{f} d(a v)+d \kappa^{f}(a v)$ for $a \in A$ and $v \in V_{n}$. When $n=n_{0}$, this is easy as $d(a v)=0$ and $f(v)=d s f(v)$ :

$$
d \kappa_{n_{0}}^{f}(a v)=a d s f(v)=a f(v)=f(a v) .
$$

Inductively, suppose that the formula holds for $n-1$. Since $d v \in C_{n-1}$, we have

$$
d\left(f-\kappa^{f} d\right)(v)=f(d v)-d \kappa^{f}(d v)=\left(\kappa^{f} d\right)(d v)=0 .
$$

Using this, and the definition of $\kappa_{n}^{f}$, we compute:

$$
\left(d \kappa_{n}^{f}\right)(v)=d s\left(f-\kappa^{f} d\right)(v)=(1-s d)\left(f-\kappa^{f} d\right)(v)=\left(f-\kappa^{f} d\right)(v) .
$$

Since $\kappa^{f} d(a v)=\kappa^{f}(a d v)=a \kappa^{f}(d v)$ by construction,

$$
d \kappa^{f}(a v)+\kappa^{f} d(a v)=a d \kappa^{f}(v)+a \kappa^{f}(d v)=a f(v)=f(a v) .
$$


Lemma 3.1.2. There is a sequence of $\mathrm{H}$-linear maps $\Upsilon^{\prime n}: \mathrm{E}(\mathrm{H}) \rightarrow \mathrm{E}(\mathrm{H})[2 n]$, starting with $\Upsilon^{\prime 0}=1$, such that $B^{\prime}\left(\Upsilon^{\prime n} \partial^{\prime}-\partial^{\prime} \Upsilon^{\prime n}\right)=0$.

They induce maps on the normalized complexes $\Upsilon^{\prime n}: H H\left(\mathrm{M}^{\prime}\right) \rightarrow H H\left(\mathrm{M}^{\prime}\right)[2 n]$.

Proof. Inductively, we suppose we have constructed $\Upsilon^{\prime n}$ satisfying $B^{\prime}\left[\Upsilon^{\prime n}, \partial^{\prime}\right]=0$. Now any chain map from $C=\mathrm{E}(\mathrm{H})$ to $\mathrm{E}(\mathrm{H})[2 n+1]$ must land in the good truncation $D=\mathrm{E}(\mathrm{H})[2 n+1]_{\geq 0}$, and the $k$-linear chain contraction $-s$ of (2.1.2) is also a contraction of $D$. We claim that the $\mathrm{H}$-linear map $f=-B^{\prime} \Upsilon^{\prime n}: C \rightarrow D$ is a chain map. Since the differential on $D$ is $-\partial^{\prime}$, the claim follows from:

$$
f\left(\partial^{\prime}\right)-\left(-\partial^{\prime}\right) f=-B^{\prime} \Upsilon^{\prime n} \partial^{\prime}-\partial^{\prime} B^{\prime} \Upsilon^{\prime n}=B^{\prime}\left(\partial^{\prime} \Upsilon^{\prime n}-\Upsilon^{\prime n} \partial^{\prime}\right)=0 .
$$

We define $\Upsilon^{\prime n+1}$ to be the $\mathrm{H}$-linear chain contraction of $f=-B^{\prime} \Upsilon^{\prime n}$ given by the formulas in Lemma 3.1.1. That is,

$$
\Upsilon^{\prime n+1}:=\kappa^{f}=\kappa^{-B^{\prime} \Upsilon^{\prime n}} \quad(n \geq 0) .
$$

The chain contraction condition $\left[\Upsilon^{\prime n+1}, \partial^{\prime}\right]=f$ for (3.1.3) implies that the inductive hypothesis $B^{\prime}\left[\Upsilon^{\prime n+1}, \partial^{\prime}\right]=B^{\prime} f=0$ holds.

Finally, note that the normalized mixed complex $H H\left(M^{\prime}\right)$ is a quotient of $\mathrm{E}(\mathrm{H})$, and its terms have the form $H H\left(M^{\prime}\right)_{n}=\mathrm{H} \otimes W_{n}$ for a quotient module $W_{n}$ of $V_{n}$. By naturality of $\kappa^{f}$ in $f$, the above construction also goes through with $\mathrm{E}(\mathrm{H})$ replaced by $M^{\prime}(\mathrm{H})$, and the maps $\Upsilon^{\prime}$ on $\mathrm{E}(\mathrm{H})$ and $H H\left(M^{\prime}\right)$ are compatible.

We define maps $\Upsilon^{\prime}: H H(\mathrm{E}(\mathrm{H})) \rightarrow H N(\mathrm{E}(\mathrm{H}))$ and $\Upsilon^{\prime}: H H\left(\mathrm{M}^{\prime}\right) \rightarrow H N\left(\mathrm{M}^{\prime}\right)$ by

$$
\Upsilon^{\prime}=\sum_{n=0}^{\infty} \Upsilon^{\prime n}
$$

That is, $\Upsilon^{\prime}(x)$ is $\left(\cdots, \Upsilon^{\prime n}(x), \cdots, \Upsilon^{\prime 1}(x), x\right)$.

Lemma 3.1.5. The maps $\Upsilon^{\prime}$ in (3.1.4) are morphisms of chain $\mathrm{H}$-modules, and $\pi^{\prime} \Upsilon^{\prime}=1$. Here $\pi^{\prime}$ is the appropriate canonical projection, either $H N(\mathrm{E}(\mathrm{H})) \rightarrow H H(\mathrm{E}(\mathrm{H}))$ or $H N\left(\mathrm{M}^{\prime}\right) \rightarrow H H\left(\mathrm{M}^{\prime}\right)$.

Proof. It is clear that $\Upsilon^{\prime}$ is H-linear and that $\pi^{\prime} \Upsilon^{\prime}=\Upsilon^{\prime 0}=1$. To see that it is a chain map, we observe that the $n$th coordinate of $\left(B^{\prime}+\partial^{\prime}\right) \Upsilon^{\prime}-\Upsilon^{\prime} \partial^{\prime}$ is $B^{\prime} \Upsilon^{\prime n-1}+\partial^{\prime} \Upsilon^{\prime n}-\Upsilon^{\prime n} \partial^{\prime}$. This is zero by the chain contraction condition for (3.1.3).

Remark 3.1.6. Write $[1]^{n}$ for the element $1 \otimes \cdots \otimes 1$ of $k^{\otimes n}$. By induction, we may check that $\Upsilon^{\prime n}(1)=(-1)^{n}(2 n) ! / n ![1]^{2 n+1}$. Thus $\Upsilon^{\prime}(1)=(0, \ldots, 0,1)$ in $H N\left(M^{\prime}\right)$.

Recall from Definition 2.4.5 that $M=k \otimes_{\mathrm{H}} M^{\prime}$, and that $\mathrm{B}(A)=k \otimes_{A} \mathrm{E}(A)$.

Corollary 3.1.7. There are morphisms of chain k-modules, $\Upsilon: H H(\mathrm{~B}(\mathrm{H})) \rightarrow H N(\mathrm{~B}(\mathrm{H}))$ and $\Upsilon: H H(\mathrm{M}) \rightarrow H N(\mathrm{M})$, defined by

$$
\Upsilon=\sum_{n=0}^{\infty} 1_{k} \otimes_{\mathbf{H}} \Upsilon^{\prime n}
$$

and $\pi \Upsilon=1$. Here $\pi$ is the appropriate projection $\pi: H N \rightarrow H H$.

\subsection{The lift $H H(\mathrm{~B}(\mathrm{H})) \stackrel{c}{\longrightarrow} H N(\mathrm{H})$}

Recall that $C(\mathrm{H})$ denotes the canonical cyclic complex of the algebra underlying $\mathrm{H}([7,2.5 .4])$. We set $\tau_{0}=\eta: k \rightarrow \mathrm{H}$.

Lemma 3.2.1. Let $\mathrm{H}$ be a cocommutative Hopf algebra. Then the $k$-linear map

$$
\begin{gathered}
\tau: \mathrm{B}(\mathrm{H}) \rightarrow C(\mathrm{H}) \\
\tau\left(h_{1} \otimes \cdots \otimes h_{n}\right)=S\left(h_{1}^{(0)} \ldots h_{n}^{(0)}\right) \otimes h_{1}^{(1)} \otimes \cdots \otimes h_{n}^{(1)}, \quad n>0,
\end{gathered}
$$

is an injective homomorphism of cyclic $k$-modules. It induces an injection of the associated mixed complexes, $M(\mathrm{H}) \hookrightarrow C(\mathrm{H})_{\text {norm }}$. 
Proof. One has to check that $\tau$ commutes with the face, degeneracy and cyclic operators; these are all straightforward, short calculations. The fact that the maps are injective follows from the antipode identity $\left(S h^{(0)}\right) h^{(1)}=\eta \epsilon(h)$.

Remark 3.2.2. If $g_{1}, \ldots, g_{n} \in \mathrm{H}$ are grouplike, then

$$
\tau\left(g_{1} \otimes \cdots \otimes g_{n}\right)=\left(g_{1} \ldots g_{n}\right)^{-1} \otimes g_{1} \otimes \cdots \otimes g_{n} .
$$

Thus for $\mathrm{H}=k[G]$, the $\tau$ of 3.2 .1 is the map $k[\mathrm{~B}(G, 1)] \hookrightarrow H H(k[G])$ of $[7,7.4 .5]$.

We define $c: \mathrm{B}(\mathrm{H}) \rightarrow H N(\mathrm{H})$ to be the natural chain map

$$
c: \mathrm{B}(\mathrm{H}) \stackrel{r}{\longrightarrow} H N(\mathrm{~B}(\mathrm{H})) \stackrel{\tau}{\longrightarrow} H N(\mathrm{H}) .
$$

We will also write $c$ for the normalized version $H H(M) \rightarrow H N(\mathrm{H})_{\text {norm }}$ of this map.

Theorem 3.2.4. The following diagram commutes

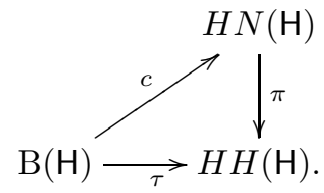

Proof. By (3.2.3), Lemma 3.2.1 and Corollary 3.1.7, $\pi c=\pi \tau \Upsilon=\tau \pi \Upsilon=\tau$.

Remark 3.2.5. Goodwillie proved in [4, II.3.2] that, up to chain homotopy, there is a unique chain map $\mathrm{B}(k[G]) \rightarrow H N(k[G])$ lifting $\tau$, natural in the group $G$. Ginot [3] has given explicit formulas for one such map; it follows that Ginot's map is naturally chain homotopic to the map $c$ constructed in (3.2.3) for $\mathrm{H}=k[G]$.

\subsection{Passage to completion}

If $A$ is a filtered algebra, the induced filtration (2.5.1) on the canonical cyclic module $C(A)$ makes it a cyclic filtered module. Passing to completion we obtain a cyclic module $C^{\text {top }}(\hat{A})$ with $C_{n}^{\text {top }}(\hat{A})=$ $\hat{A}^{\otimes}{ }^{\otimes n+1}$. In the spirit of subsection 2.5 , we write $H H^{\text {top }}(\hat{A}), H N^{\text {top }}(\hat{A})$, etc. for the Hochschild and cyclic complexes etc. of the mixed complex associated to $C^{\text {top }}(\hat{A})$.

In particular this applies if $A=\mathrm{H}$ is a cocommutative Hopf algebra, equipped with an $I$-adic filtration, where $I$ is an ideal and coideal of $\mathrm{H}$ with $\epsilon(I)=0$, closed under the antipode $S$. Write $\hat{\mathrm{H}}$ for the associated complete Hopf algebra.

It is clear from the formula in Lemma 3.2.1 that $\tau$ is a morphism of cyclic filtered modules. Hence it induces continuous maps $\hat{\tau}$ between the corresponding complexes for $H H, H N$, etc.

Propostion 3.3.1. The map $c$ of (3.2.3) induces a continuous map $\hat{c}$ which fits into a commutative diagram

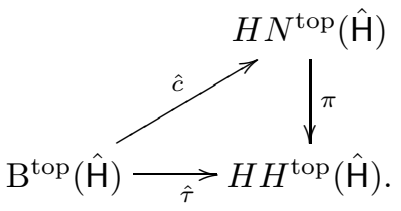

Proof. It suffices to show that $\Upsilon$ (and hence $c$ ) is a filtered morphism. We observed in 2.5 that $\mathrm{E}(\mathrm{H})$ is a cyclic filtered module. Similarly, it is clear that $s, s^{\prime}$ and $B^{\prime}$ are filtered morphisms from their definitions in (2.1.2), (2.4.3) and (2.4.4). The recursion formulas in Lemma 3.1.1 and (3.1.3) show that each $\Upsilon^{\prime n}$ is filtered, whence so are $\Upsilon^{\prime}$ and $\Upsilon$, as required. 


\section{The case of universal enveloping algebras of Lie algebras}

Let $\mathfrak{g}$ be a Lie algebra over a commutative ring $k$. Then the enveloping algebra $U \mathfrak{g}$ is a cocommutative Hopf algebra, so the constructions of the previous sections apply to $U \mathfrak{g}$. In particular a natural map $c: \mathrm{B}(U \mathfrak{g}) \rightarrow H N(U \mathfrak{g})$ was constructed in (3.2.3). In this section, we show that the Loday-Quillen map

$$
\wedge \mathfrak{g} \stackrel{\theta}{\longrightarrow} C^{\lambda}(U \mathfrak{g})[-1] \stackrel{B}{\longrightarrow} H N(U \mathfrak{g})
$$

factors through $c$ up to chain homotopy. (See Theorem 4.2.2.)

\subsection{Chevalley-Eilenberg complex}

The Chevalley-Eilenberg resolution of $k$ as a $U \mathfrak{g}$-module has the form $\left(U \mathfrak{g} \otimes \wedge \mathfrak{g}, d^{\prime}\right)$, and is given in $[13,7.7]$. Tensoring it over $U \mathfrak{g}$ with $k$, we obtain a complex $(\wedge \mathfrak{g}, d)$. Kassel showed in $[6,8.1]$ that the anti-symmetrization map

$$
\begin{aligned}
e & : \wedge^{n} \mathfrak{g} \rightarrow(U \mathfrak{g})^{\otimes n} \\
e\left(x_{1} \wedge \cdots \wedge x_{n}\right) & =\sum_{\sigma \in \mathfrak{S}_{n}} \operatorname{sg}(\sigma) x_{\sigma(1)} \otimes \cdots \otimes x_{\sigma(n)}
\end{aligned}
$$

induces chain maps $e: \wedge \mathfrak{g} \rightarrow \mathrm{B}(U \mathfrak{g})$ and $1 \otimes e: U \mathfrak{g} \otimes \wedge \mathfrak{g} \rightarrow \mathrm{E}(U \mathfrak{g})$, because $e d=\partial e$ and $(1 \otimes e) d^{\prime}=\partial^{\prime}(1 \otimes e)$. Moreover, $e$ and $1 \otimes e$ are quasi-isomorphisms; see [6, 8.2].

Lemma 4.1.2. The map $\psi^{\prime}: U \mathfrak{g} \otimes \wedge \mathfrak{g} \rightarrow H N\left(\mathrm{M}^{\prime}(U \mathfrak{g})\right)$ defined by

$$
\psi^{\prime}(x)=(\ldots, 0,0,0,1 \otimes e(x))
$$

is a morphism of chain $U \mathfrak{g}$-modules, and the map $\psi: \wedge \mathfrak{g} \rightarrow H N(\mathrm{M}(U \mathfrak{g}))$ defined by

$$
\psi(x)=(\ldots, 0,0,0, e(x))
$$

is a morphism of chain $k$-modules.

Proof. Consider $U \mathfrak{g} \otimes \wedge \mathfrak{g}$ and $\wedge \mathfrak{g}$ as mixed complexes with trivial Connes' operator. By Corollary 2.4.8, $B^{\prime}(1 \otimes e)=0$ in $M^{\prime}(U \mathfrak{g})$. Thus both $1 \otimes e$ and $e$ induce morphisms of mixed complexes $U \mathfrak{g} \otimes \wedge \mathfrak{g} \rightarrow \mathrm{M}^{\prime}(U \mathfrak{g})$ and $\wedge \mathfrak{g} \rightarrow \mathrm{M}^{\prime}(U \mathfrak{g})$

Lemma 4.1.3. The diagrams
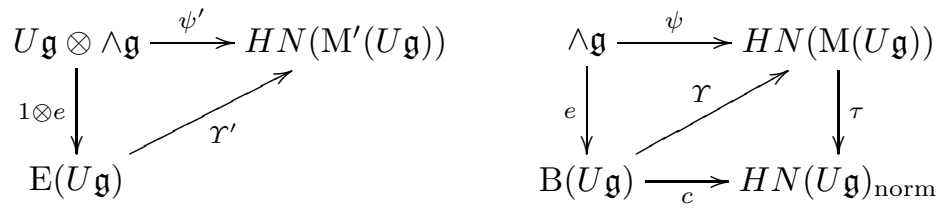

commute up to natural Ug-linear (resp., natural $k$-linear) chain homotopy.

Proof. By 3.1.7, 4.1.2 and (3.2.3), it suffices to consider the left diagram.

Consider the mixed subcomplex $N \subset \mathrm{M}^{\prime}(U \mathfrak{g})$ given by $N_{0}=0, N_{1}=\operatorname{ker} \partial^{\prime}$ and $N_{n}=\mathrm{E}_{n}(U)_{\text {norm }}$. Because $\psi^{\prime}(1)=\Upsilon^{\prime}(1)$ by Example 3.1.6, the difference $f=\Upsilon^{\prime}(1 \otimes e)-\psi^{\prime}$ factors through $H N(N)$. Put $\phi^{n}=(-1)^{n}\left(s B^{\prime}\right)^{n} s: N \rightarrow N[2 n+1]$. One checks that $\phi:=\sum_{i=0}^{\infty} \phi^{n}$ is a natural, $k$-linear contracting homotopy for $H N(N)$. Now apply Lemma 3.1.1. 
There are simple formulas for $\tau$ and $\tau \psi$ in the normalized complexes.

Lemma 4.1.4. Let $x_{1}, \ldots, x_{n} \in \mathfrak{g}$. Then in $C(U \mathfrak{g})_{\text {norm }}$ we have:

$$
\tau\left(x_{1} \otimes \cdots \otimes x_{n}\right)=1 \otimes x_{1} \otimes \cdots \otimes x_{n}
$$

Proof. Let $\nabla^{(n)}: U \mathfrak{g}^{\otimes n} \rightarrow U \mathfrak{g}$ be the multiplication map and $\sigma \in \mathfrak{S}_{2 n}$ the (bad) riffle shuffle $\sigma(2 i-1)=i, \sigma(2 i)=n+i$. By definition (see 3.2.1),

$$
\tau=\left(S \otimes 1^{\otimes n}\right) \circ\left(\nabla^{(n)} \otimes 1^{\otimes n}\right) \circ \sigma \circ \Delta^{\otimes n}
$$

in $C(U \mathfrak{g})$. Since the $x_{i}$ are primitive,

$$
\Delta^{\otimes n}\left(x_{1} \otimes \cdots \otimes x_{n}\right)=\left(x_{1} \otimes 1+1 \otimes x_{1}\right) \otimes \cdots \otimes\left(x_{n} \otimes 1+1 \otimes x_{n}\right)
$$

Expanding this product gives a sum in which

$$
x=1 \otimes x_{1} \otimes 1 \otimes x_{2} \otimes \cdots \otimes 1 \otimes x_{n}
$$

is the only term not mapped to a degenerate element of $C(U \mathfrak{g})$ under the composition (4.1.5). Thus in $C(U \mathfrak{g})_{\text {norm }}$ we have:

$$
\begin{aligned}
& \tau\left(x_{1} \otimes \cdots \otimes x_{n}\right)=\left(S \otimes 1^{\otimes n}\right)\left(\nabla^{(n)} \otimes 1^{\otimes n}\right) \sigma(x) \\
= & \left(S \otimes 1^{\otimes n}\right)\left(\nabla^{(n)} \otimes 1^{\otimes n}\right)\left(1 \otimes \cdots \otimes 1 \otimes x_{1} \otimes \cdots \otimes x_{n}\right) \\
= & \left(S \otimes 1^{\otimes n}\right)\left(1 \otimes x_{1} \otimes \cdots \otimes x_{n}\right)=1 \otimes x_{1} \otimes \cdots \otimes x_{n} .
\end{aligned}
$$

Corollary 4.1.6. We have: $\tau \psi\left(x_{1} \wedge \cdots \wedge x_{n}\right)=\left(\ldots, 0,0,0,1 \otimes e\left(x_{1} \wedge \cdots \wedge x_{n}\right)\right)$.

Proof. Combine Lemmas 4.1.2 and 4.1.4.

\subsection{The Loday-Quillen map}

We can now show that $\tau \psi$ factors through the Connes' complex $C^{\lambda}(U \mathfrak{g})=\operatorname{coker}\left(1-t: U^{\otimes *} \rightarrow\right.$ $H H(U \mathfrak{g}))$. We have a homomorphism $\theta$ which lifts the Loday-Quillen map of [7, 10.2.3.1, 11.3.12] to $U \mathfrak{g}:$

$$
\begin{gathered}
\theta: \wedge^{n+1} \mathfrak{g} \rightarrow C_{n}^{\lambda}(U \mathfrak{g}) \\
\theta\left(x_{0} \wedge x_{1} \wedge \cdots \wedge x_{n}\right)=x_{0} \otimes e\left(x_{1} \wedge \cdots \wedge x_{n}\right) .
\end{gathered}
$$

Because we are working modulo the image of $1-t, \theta$ is well defined. The following result is implicit in the proof of $[7,10.2 .4]$ for $r=1, A=U \mathfrak{g}$.

Lemma 4.2.1. $\theta$ is a chain homomorphism $\wedge \mathfrak{g} \rightarrow C^{\lambda}(U \mathfrak{g})[-1]$.

Proof. To show that $b \theta=-\theta d$, we fix a monomial $x_{1} \wedge \cdots \wedge x_{n}$ and compute that $b \theta\left(x_{0} \wedge \cdots \wedge x_{n}\right)=$ $\sum_{\sigma \in \mathfrak{S}_{n}} b\left(x_{0} \otimes x_{\sigma(1)} \otimes \cdots \otimes x_{\sigma(n)}\right)=A+B$, where $A$ equals:

$$
\begin{gathered}
\sum_{\sigma \in \mathfrak{S}_{n}} \operatorname{sg}(\sigma)\left(x_{0} x_{\sigma(1)} \otimes x_{\sigma(2)} \otimes \cdots \otimes x_{\sigma(n)}+(-1)^{n} x_{\sigma(n)} x_{0} \otimes x_{\sigma(1)} \otimes \cdots \otimes x_{\sigma(n-1)}\right) \\
=\sum_{\sigma \in \mathfrak{S}_{n}} \operatorname{sg}(\sigma)\left(x_{0} x_{\sigma(1)} \otimes x_{\sigma(2)} \otimes \cdots \otimes x_{\sigma(n)}-x_{\sigma(1)} x_{0} \otimes x_{\sigma(2)} \otimes \cdots \otimes x_{\sigma(n)}\right) \\
=\sum_{i=1}^{n} \sum_{\substack{\sigma \in \mathfrak{G} \\
\sigma(i)=i}}(-1)^{i-1} \operatorname{sg}(\sigma)\left[x_{0}, x_{i}\right] \otimes x_{\sigma(1)} \otimes \cdots \otimes x_{\sigma(i-1)} \otimes x_{\sigma(i+1)} \otimes \cdots \otimes x_{\sigma(n)} \\
=\sum_{i=1}^{n}(-1)^{i-1}\left[x_{0}, x_{i}\right] \otimes e\left(x_{1} \wedge \cdots \wedge x_{i-1} \wedge x_{i+1} \wedge \cdots \wedge x_{n}\right),
\end{gathered}
$$


and $B$ equals

$$
\begin{gathered}
\sum_{\sigma \in \mathfrak{S}_{n}} \sum_{i=1}^{n-1}(-1)^{i} \operatorname{sg}(\sigma) x_{0} \otimes x_{\sigma(1)} \otimes \cdots \otimes x_{\sigma(i)} x_{\sigma(i+1)} \otimes \cdots \otimes x_{\sigma(n)} \\
=x_{0} \otimes e\left(d\left(x_{1} \wedge \cdots \wedge x_{n}\right)\right) .
\end{gathered}
$$

Similarly, $\theta d\left(x_{0} \wedge \cdots \wedge x_{n}\right)$ is the sum of $-A$ and

$$
\sum_{0<i<j \leq n}(-1)^{i+j+1} x_{0} \otimes e\left(\left[x_{i}, x_{j}\right] \wedge x_{1} \wedge \cdots \wedge x_{n}\right)=-x_{0} \otimes e\left(d\left(x_{1} \wedge \cdots \wedge x_{n}\right)\right)
$$

which equals $-B$. Therefore $b \theta\left(x_{0} \wedge \cdots \wedge x_{n}\right)=-\theta d\left(x_{0} \wedge \cdots \wedge x_{n}\right)$.

Warning: the sign convention used for $d$ in $[7,10.1 .3 .3]$ differs by -1 from the usual convention, used here and in $[13,7.7]$ and $[6]$.

It is well known and easy to see that, because Connes' operator $B$ vanishes on the image of $1-t$, it induces a chain map for every algebra $A$ :

$$
\begin{gathered}
B: C^{\lambda}(A)[-1] \rightarrow H N(A) \\
B[x]=(\ldots, 0,0,0, B x) .
\end{gathered}
$$

Let $\wedge^{+} \mathfrak{g}$ denote the positive degree part of $\wedge \mathfrak{g}$.

Theorem 4.2.2. We have $\tau \psi=B \theta$ as chain maps $\wedge^{+} \mathfrak{g} \rightarrow H N(U \mathfrak{g})_{\text {norm }}$.

Hence the following diagram commutes up to natural chain homotopy.

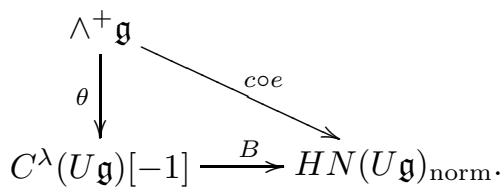

Theorem 4.2.2 fails for the degree 0 part $k$ of $\wedge \mathfrak{g}$. Indeed, $\theta(1)=0$ for degree reasons, while from Example 3.1.6 we see that $c e(1)=\tau \psi(1)=(\ldots, 0,1)$ is nonzero.

Proof. By Lemma 4.1.3, it suffices to check that $\tau \psi=B \theta$. By Corollary 4.1.6 and (4.1.1), we have

$$
\tau \psi\left(x_{1} \wedge \cdots \wedge x_{n}\right)=\left(\ldots, 0,0, \sum_{\sigma \in \mathfrak{S}_{n}} \operatorname{sg}(\sigma) 1 \otimes x_{\sigma(1)} \otimes \cdots \otimes x_{\sigma(n)}\right) .
$$

Note the expression above contains no products in $U \mathfrak{g}$; neither do the formulas for $\theta\left(x_{0} \wedge x_{1} \wedge \cdots \wedge x_{n}\right)$ and the Connes' operator in $C(U \mathfrak{g})$. Thus we may assume that $\mathfrak{g}$ is abelian, and that $U \mathfrak{g}=S \mathfrak{g}$ is a (commutative) symmetric algebra. We may interpret $\theta\left(x_{1} \wedge \cdots \wedge x_{n}\right)$ as the shuffle product $x_{1} \star B\left(x_{2}\right) \star \cdots \star B\left(x_{n}\right)$ (see $[7,4.2 .6]$ ), and the nonzero coordinate of (4.2.3) as the shuffle product

$$
\begin{array}{cc}
\left(1 \otimes x_{1}\right) \star \cdots \star\left(1 \otimes x_{n}\right)=B\left(x_{1}\right) \star \cdots \star B\left(x_{n}\right) \\
=B\left(x_{1} \star B\left(x_{2}\right) \star \cdots \star B\left(x_{n}\right)\right) & \text { by }[8,3.1] \text { or }[7,4.3 .5] \\
=B\left(\theta\left(x_{1} \wedge \cdots \wedge x_{n}\right)\right) . & \square
\end{array}
$$




\section{Nilpotent Lie algebras and nilpotent groups}

In this and the remaining sections we shall fix the ground $\operatorname{ring} k=\mathbb{Q}$. Let $\mathfrak{g}$ be a nilpotent Lie algebra; consider the completion $\hat{U} \mathfrak{g}$ of its enveloping algebra with respect to the augmentation ideal, and set

$$
G=\exp \mathfrak{g} \subset \hat{U} \mathfrak{g} .
$$

This is a nilpotent group, and $\mathrm{H}=\mathbb{Q}[G]$ is a Hopf algebra. The inclusion $G \subset \hat{U} \mathfrak{g}$ induces a homomorphism $\mathrm{H}=\mathbb{Q}[G] \hookrightarrow \hat{U} \mathfrak{g}$, and $\hat{\mathrm{H}} \cong \hat{U} \mathfrak{g}$ by $[10, \mathrm{~A} .3]$.

On the other hand, Suslin and Wodzicki showed in $[12,5.10]$ that there is a natural quasiisomorphism $s w: \mathrm{B}(\mathbb{Q}[G]) \rightarrow \wedge \mathfrak{g}$. Putting these maps together with those considered in the previous sections, we get a diagram

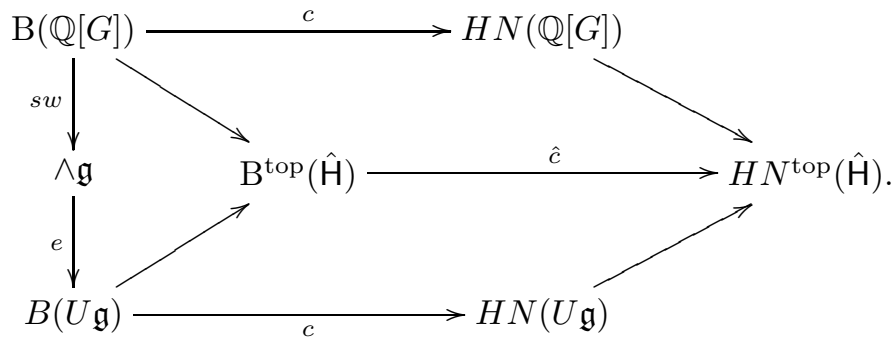

Propostion 5.2. Diagram (5.1) commutes up to natural chain homotopy.

Proof. The two parallelograms commute by naturality. The triangle on the left of (5.1) commutes up to natural homotopy by Lemma 5.3 below.

Lemma 5.3. The following diagram commutes up to natural chain homotopy.

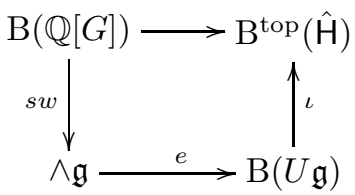

Proof. By construction (see $[12,5.10]$ ), the map $s w$ is induced by a map $\mathrm{E}(\mathbb{Q}[G]) \rightarrow \hat{\mathrm{H}} \hat{\otimes} \wedge \mathfrak{g}$. Let $\iota$ be the upward vertical map; $\iota e$ is induced by $1 \otimes \iota e: \hat{\mathrm{H}} \hat{\otimes} \wedge \mathfrak{g} \rightarrow \mathrm{E}^{\text {top }}(\hat{\mathrm{H}})$. Thus it suffices to show that the composite $\mathrm{E}(\mathbb{Q}[G]) \rightarrow \mathrm{E}^{\mathrm{top}}(\hat{\mathrm{H}})$ is naturally homotopic to the map induced by the homomorphism $\mathbb{Q}[G] \rightarrow \hat{\mathrm{H}}$. By definition, these maps agree on $\mathrm{E}_{0}(\mathbb{Q}[G])$; thus their difference goes to the subcomplex $\operatorname{ker}\left(\hat{\epsilon}: \mathrm{E}^{\mathrm{top}}(\hat{\mathrm{H}}) \rightarrow \mathbb{Q}\right)$ which is contractible, with contracting homotopy induced by (2.1.2). Hence we may apply Lemma 3.1.1; this finishes the proof.

Remark 5.4. Let $\mathfrak{g}=J_{\text {Lie }}$ be the Lie algebra associated to an ideal $J$ in a $\mathbb{Q}$-algebra $A$ ( $\mathfrak{g}$ is $J$ with the commutator bracket); there is a canonical algebra map $U \mathfrak{g} \rightarrow A$ sending $\mathfrak{g}$ onto $J$. If $J$ is a nilpotent ideal then $\mathfrak{g}$ is a nilpotent Lie algebra and the induced algebra map $\hat{U} \mathfrak{g} \rightarrow A$ restricts to an isomorphism $G=\exp (\mathfrak{g}) \stackrel{\cong}{\rightrightarrows}(1+J)^{\times}$, as is proven in $[12,5.2]$.

Let $C(A, J)$ denote the kernel of $C(A) \rightarrow C(A / J)$, and let $C^{\lambda}(U \mathfrak{g}, \mathcal{I})$ denote the kernel of $C^{\lambda}(U \mathfrak{g}) \rightarrow C^{\lambda}(k)$. The composite of $\theta$ with the map induced by $U \mathfrak{g} \rightarrow A$ factors through $C^{\lambda}(A, J)$, giving rise to a commutative diagram

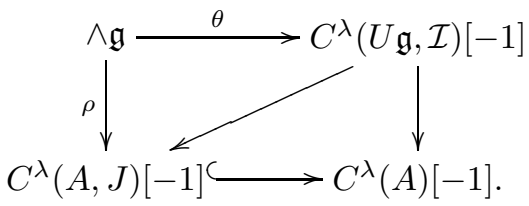


The composite $\mathbb{Q}[G] \rightarrow \hat{U} \mathfrak{g} \rightarrow A$ sends the augmentation ideal $\mathcal{I}_{G}$ of $\mathbb{Q}[G]$ to $J$. Consider the resulting map

$$
j: H N\left(\mathbb{Q}[G], \mathcal{I}_{G}\right) \rightarrow H N(\hat{U} \mathfrak{g}, \hat{\mathcal{I}}) \rightarrow H N(A, J) .
$$

Putting together Theorem 4.2.2 with Proposition 5.2, we get a naturally homotopy commutative diagram (with $G=(1+J)^{\times}$):

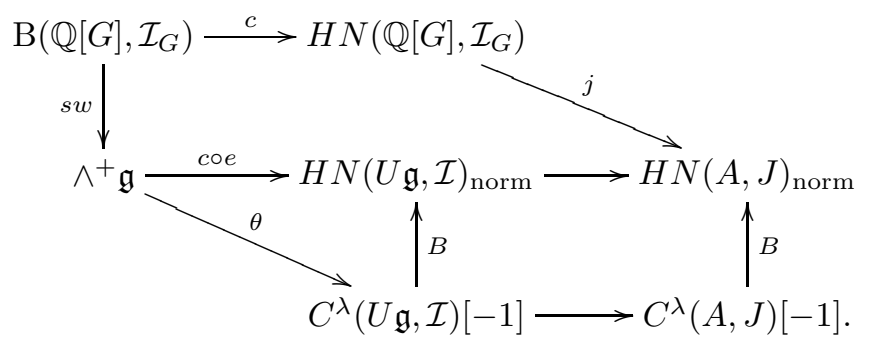

\section{The relative Chern character of a nilpotent ideal}

In this section we establish Theorem 6.5.1, promised in (1.3), that the two definitions (1.1) and (1.2) of the Chern character $K_{*}(A, I) \rightarrow H N_{*}(A, I)$ agree for a nilpotent ideal $I$ in a unital $\mathbb{Q}$ algebra $A$. The actual proof is quite short, and most of this section is devoted to the construction of the maps (1.1) and (1.2).

For this it is appropriate to regard a non-negative chain complex $C$ as a simplicial abelian group via Dold-Kan, identifying the homology of the complex with the homotopy groups $\pi_{*}(C)$ by abuse of notation. If $X$ is a simplicial set, we write $\mathbb{Z}[X]$ for its singular complex, so that $H_{*}(X ; \mathbb{Z})$ is $\pi_{*} \mathbb{Z}[X]$, and the Hurewicz map is induced by the simplicial map $h: X \rightarrow \mathbb{Z}[X]$.

\subsection{The absolute Chern character}

Let $A$ be a unital $\mathbb{Q}$-algebra, and $\operatorname{BGL}(A)$ the classifying space of $G L(A)$. Now the plus construction $\mathrm{BGL}(A) \rightarrow \operatorname{BGL}(A)^{+}$is a homology isomorphism, and $K_{n}(A)=\pi_{n} \mathrm{BGL}(A)^{+}$for $n \geq 1$. In particular, the singular chain complex map $\mathbb{Z}[\operatorname{BGL}(A)] \rightarrow \mathbb{Z}\left[\operatorname{BGL}(A)^{+}\right]$is a quasi-isomorphism. As described in [7, 11.4.1], the absolute Chern character $c h_{n}: K_{n}(A) \rightarrow H N_{n}(A)$ (of Goodwillie, Jones et al.) is the composite $c h=c h_{A}^{-} \circ h$ of the Hurewicz map $h: \mathrm{B} G L(A)^{+} \rightarrow \mathbb{Z}\left[\mathrm{B} G L(A)^{+}\right] \simeq$ $\mathbb{Z}[\operatorname{BGL}(A)]$, the identification $\mathbb{Z}[\mathrm{B} G]=\mathrm{B}(\mathbb{Z}[G])$ with the bar complex, and the chain complex map $c h_{A}^{-}$, which is defined as the stabilization (for $G L_{n} \subset G L_{n+1}$ ) of the composites:

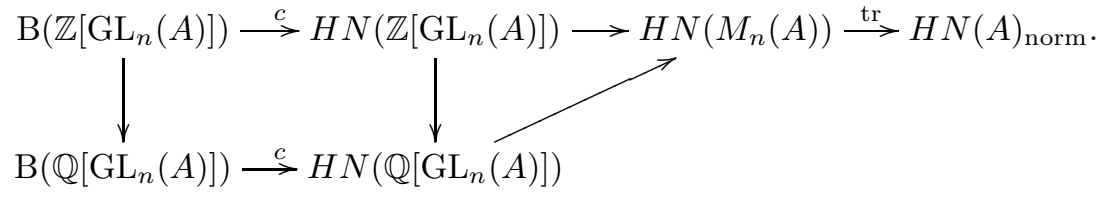

Here $c$ is the natural map defined in (3.2.3) for $k=\mathbb{Z}$ and $\mathbb{Q}$; the middle maps in the diagram are induced by the fusion maps $\mathbb{Z}\left[\mathrm{GL}_{n}(A)\right] \subset \mathbb{Q}\left[\mathrm{GL}_{n}(A)\right] \rightarrow M_{n}(A)$, and $\operatorname{tr}$ is the trace map. The maps $H N\left(\mathbb{Q}\left[\mathrm{GL}_{n}(A)\right]\right) \rightarrow H N(A)_{\text {norm }}$ are independent of $n$ by $[7,8.4 .5]$, even though the fusion maps are not.

Remark 6.1.2. If $A$ is commutative and connected, the composition of the rank map $K_{0}(A) \rightarrow \mathbb{Z}$ sending $\left[A^{r}\right]$ to $r$ with the map $H_{0}\left(c h_{A}^{-}\right)$yields the Chern character $c h_{0}^{-}: K_{0}(A) \rightarrow H N_{0}(A)$ of $[7,8.3]$. Composing this with the maps $H N(A) \rightarrow H C(A)[2 n]$ yields the map $c_{0, n}: K_{0}(A) \rightarrow$ $H C_{2 n}(A)$ of $[7,8.3 .4]$. From Example 3.1.6 above, with $A=k$, we see that $\operatorname{ch}([k])=c(1)$, and $\operatorname{ch}_{0, n}([k])=(-1)^{n}(2 n) ! / n !$ in $H C_{2 n}(k) \cong k$, in accordance with $[7,8.3 .7]$. 


\subsection{Volodin models for the relative Chern character of nilpotent ideals}

In order to define the relative version $c h_{*}$ of the absolute Chern character, we need to recall a chunk of notation about Volodin models. For expositional simplicity, we shall assume that $I$ is a nilpotent ideal in a unital $\mathbb{Q}$-algebra $A$.

Definition 6.2.1. Let $I$ a nilpotent ideal in a $\mathbb{Q}$-algebra $A$, and $\sigma$ a partial order of $\{1, \ldots, n\}$. We let $\mathcal{T}_{n}^{\sigma}(A, I)$ be the nilpotent subalgebra of $M_{n}(A)$ defined by:

$$
\mathcal{T}_{n}^{\sigma}(A, I):=\left\{a \in M_{n}(A): a_{i j} \in I \text { if } i \nless_{\sigma} j\right\} .
$$

We write $\mathfrak{t}_{n}^{\sigma}(A, I)$ for the associated Lie algebra, and $T_{n}^{\sigma}(A, I)$ for the group

$$
T_{n}^{\sigma}(A, I)=\exp _{n}^{\sigma}(A, I)=1+\mathcal{T}_{n}^{\sigma}(A, I) \subset G L_{n}(A) .
$$

The Volodin space $X(A) \subset B G L(A)$ is defined to be the union of the spaces $X_{n}(A)=$ $\bigcup_{\sigma} B T_{n}^{\sigma}(A, 0)$; see $[7,11.2 .13]$. The relative Volodin space $X(A, I)$ is defined to be the union of the spaces $\bigcup_{\sigma} B T_{n}^{\sigma}(A, I)$; see $[7,11.3 .3]$.

The morphism $c h_{A}^{-}: \mathbb{Q}[B \mathrm{GL}(A)] \rightarrow H N(A)_{\text {norm }}$ of (6.1.1) sends the subcomplex $\mathbb{Q}[X(A, I)]$ to $H N(A, I)_{\text {norm }}$, which is the kernel of $H N(A)_{\text {norm }} \rightarrow H N(A / I)_{\text {norm }}$; see [7, 11.4.6]. The chain map $c h^{-}$is defined to be the restriction of $c h_{A}^{-}$:

$$
c h^{-}: \mathbb{Q}[X(A, I)] \rightarrow H N(A, I)_{\text {norm }} .
$$

It will be useful to have a more detailed description of the restriction of $(6.2 .2)$ to $\mathbb{Q}\left[B T_{n}^{\sigma}(A, I)\right]$. Recall that if $\Lambda$ is a non-unital subalgebra of $R$ then $\mathbb{Q}+\Lambda$ is a unital subalgebra of $R$; we write $C(\Lambda)$ for the cyclic submodule $C(\mathbb{Q}+\Lambda, \Lambda)$ of $C(\mathbb{Q}+\Lambda)$ and hence of $C(R)$, following [7, 2.2.16]. When $\Lambda=\mathcal{T}_{n}^{\sigma}(A, I)$ and $R=M_{n}(A)$, we obtain the cyclic submodule $C\left(\mathcal{T}_{n}^{\sigma}(A, I)\right)$ of $C\left(M_{n}(A)\right)$. The trace map $C\left(M_{n}(A)\right) \rightarrow C(A)$ is a morphism of cyclic modules, sending $C\left(\mathcal{T}_{n}^{\sigma}(A, I)\right)$ to the submodule $C(A, I)$. On the other hand, by Example 5.4, we also have a map

$$
C\left(\mathbb{Q}[G], \mathcal{I}_{G}\right) \stackrel{j}{\longrightarrow} C(G), \quad \text { for } G=\mathcal{T}_{n}^{\sigma}(A, I) .
$$

From the definition of $c h_{A}^{-}$in (6.1.1) and the naturality of $c$, we obtain the promised description of $c h^{-}$, which we record.

Lemma 6.2.3. Set $G=T_{n}^{\sigma}(A, I)$. The restriction of $\mathrm{ch}^{-}$to $\mathrm{B}\left(\mathbb{Q}[G], \mathcal{I}_{G}\right)$ is the composition

$$
\mathrm{B}\left(\mathbb{Q}[G], \mathcal{I}_{G}\right) \stackrel{c}{\longrightarrow} H N\left(\mathbb{Q}[G], \mathcal{I}_{G}\right) \stackrel{j}{\longrightarrow} H N\left(\mathcal{T}_{n}^{\sigma}(A, I)\right) \stackrel{\text { tr }}{\longrightarrow} H N(A, I)_{\text {norm }} .
$$

\subsection{The relative Chern character for rational nilpotent ideals}

When $I$ is a nilpotent ideal in an algebra $A$, we define $K(A, I)$ to be the homotopy fiber of $B \mathrm{GL}(A)^{+} \rightarrow B \mathrm{GL}(A / I)^{+} ; K(A, I)$ is a connected space whose homotopy groups are the relative $K$-groups $K_{n}(A, I)$ for all $n$. We now cite Theorem 6.1 of [9] for nilpotent $I$; the proof in [9] is reproduced on page 361 of [7].

Theorem 6.3.1. If I is a nilpotent ideal in $A$, there are homotopy fibrations

$$
\begin{gathered}
X(A, I) \rightarrow B \mathrm{GL}(A) \rightarrow B \mathrm{GL}(A / I)^{+}, \\
X(A) \rightarrow X(A, I) \rightarrow K(A, I) .
\end{gathered}
$$

Moreover, $X(A, I)^{+} \stackrel{\sim}{\longrightarrow} K(A, I)$ and $\mathbb{Z}[X(A, I)] \stackrel{\sim}{\longrightarrow} \mathbb{Z}[K(A, I)]$ are homotopy equivalences (i.e., $X(A, I) \rightarrow K(A, I)$ is a homology isomorphism).

Definition 6.3.2. (See [7, 11.4.7]) The relative Chern character for the ideal I of a $\mathbb{Q}$-algebra $A$ is the composite of the Hurewicz map, the inverse of the homotopy equivalence of Theorem 6.3.1 and the map $\mathrm{ch}^{-}$of (6.2.2):

$$
c h: K(A, I) \stackrel{h}{\longrightarrow} \mathbb{Q}[K(A, I)] \underset{6.3 .1}{\stackrel{\sim}{\sim}}[X(A, I)] \stackrel{c h^{-}}{\longrightarrow} H N(A, I)_{\text {norm }} .
$$




\subsection{The rational homotopy theory character for nilpotent ideals}

For a nilpotent ideal $I$, consider the chain subcomplex of the Chevalley-Eilenberg complex $\wedge \mathfrak{g l}(A)$,

$$
x(A, I)=\sum_{n, \sigma} \wedge \mathfrak{t}_{n}^{\sigma}(A, I) .
$$

Because $s w$ is natural in $G$, the family of maps $\mathrm{B}\left(\mathbb{Q}\left[T_{n}^{\sigma}(A, I)\right]\right) \stackrel{s w}{\longrightarrow} \wedge \mathfrak{t}_{n}^{\sigma}(A, I)$ induces a morphism of complexes

$$
s w_{X}: \mathbb{Q}[X(A, I)] \rightarrow x(A, I) .
$$

On the other hand, for each $n$ and $\sigma$, the composite of the map $B \rho: \wedge \mathfrak{t}_{n}^{\sigma}(A, I) \rightarrow H N\left(\mathcal{T}_{n}^{\sigma}(A, I)\right)$ of Example 5.4 with the inclusion and the trace, i.e., with

$$
H N\left(\mathcal{T}_{n}^{\sigma}(A, I)\right) \subset H N\left(M_{n}(A)\right) \stackrel{\operatorname{tr}}{\longrightarrow} H N(A),
$$

sends $\wedge \mathfrak{t}_{n}^{\sigma}(A, I)$ into the subcomplex $H N(A, I)$. All of these maps are natural in $n$ and $\sigma$; by abuse of notation, we write $\operatorname{tr}(B \rho)$ for the resulting map:

$$
\operatorname{tr}(B \rho): x(A, I) \rightarrow H N(A, I) .
$$

Definition 6.4.1. The map $c h_{\mathrm{rht}}^{-}: \mathbb{Q}[X(A, I)] \rightarrow x(A, I) \rightarrow H N(A, I)_{\text {norm }}$ is defined to be $\operatorname{tr}(B \rho) \circ s w_{X}$, followed by $H N(A, I) \rightarrow H N(A, I)_{\text {norm }}$. The rational homotopy theory character of [7, 11.3.1], cited in (1.2), is the composite $c h^{\prime}: K(A, I) \rightarrow H N(A, I)_{\text {norm }}$ defined by:

$$
K(A, I) \stackrel{h}{\longrightarrow} \mathbb{Q}(K(A, I)) \stackrel{\rightleftharpoons}{\leftarrow} \mathbb{Q}[X(A, I)] \stackrel{c h_{\text {rht }}^{-}}{\longrightarrow} H N(A, I)_{\text {norm }} .
$$

Remark 6.4.2. We will not need the unnormalized version of $c h^{\prime}$. By construction, $c h^{\prime}$ is the map $K(A, I) \rightarrow C^{\lambda}(A, I)[-1]$ of [2, A.13], followed by Connes' operator $B$.

\subsection{Main theorem}

Let $I$ be a nilpotent ideal in a $\mathbb{Q}$-algebra $A$. The relative Chern character $c h$ of Definition 6.3 .2 induces the relative Chern character $c h_{*}$ of (1.1) on homotopy groups, and the rational homotopy character $c h^{\prime}$ of Definition 6.4.1 induces the character $c h_{*}^{\prime}$ of (1.2) on homotopy groups. Therefore the equality $c h_{*}=c h_{*}^{\prime}$ of (1.3) is an immediate consequence of our main theorem.

Theorem 6.5.1. The maps $\mathrm{ch}^{-}$and $c h_{\mathrm{rht}}^{-}$are naturally chain homotopic. Hence the maps ch and $c h^{\prime}$ are homotopic for each $A$ and $I$.

Proof. We first consider the restriction of $c h^{-}$and $c h_{\text {rht }}^{-}$to $\mathrm{B}\left(\mathbb{Q}\left[T_{n}^{\sigma}(A, I)\right]\right)$ for some fixed $n$ and $\sigma$. By Lemma 6.2.3, the restriction of $c h^{-}$to $\mathrm{B}\left(\mathbb{Q}\left[T_{n}^{\sigma}(A, I)\right]\right)$ is the map $\operatorname{tr}(j c)$; by Example 5.4, there is a natural chain homotopy from $j c$ to $B \rho \circ s w$. Since $\operatorname{tr}(B \rho) \circ s w$ is the restriction of $c h_{\text {rht }}^{-}$ to $\mathrm{B}\left(\mathbb{Q}\left[T_{n}^{\sigma}(A, I)\right]\right)$, the chain homotopies glue together by naturality to give the desired chain homotopy from $c h_{\text {rht }}^{-}$to $c h^{-}$.

\subsection{Naturality}

In order to formulate a naturality result for the homotopy between $c h$ and $c h^{\prime}$, it is necessary to give definitions for the maps $c h$ and $c h^{\prime}$ which are natural in $A$ and $I$. Contemplation of Definitions 6.3.2 and 6.4.1 shows that we need to find a natural inverse for the backwards quasiisomorphism of Theorem 6.3.1. One standard way is to fix a small category of pairs $(A, I)$ (say all pairs with a fixed cardinality bound on $A$ ) and consider the global model structure on the category 
of covariant functors from this category to Simplicial Sets; this will yield naturality with respect to all morphisms in the small category.

Let $K(A, I)^{\prime}$ be the cofibrant replacement of $K(A, I)$, and factor the backwards map as $\mathbb{Q}[K(A, I)] \stackrel{\simeq}{\approx} C \stackrel{\simeq}{\leftarrow} \mathbb{Q}[X(A, I)]$. Then $h$ lifts to a map $h^{\prime}: K(A, I)^{\prime} \rightarrow C$ and, since $H N(A, I)$ is fibrant, $c h^{-}$and $c h_{\text {rht }}^{-}$both lift to maps $C \rightarrow H N(A, I)$. We can then define $c h$ to be the

composite $K(A, I)^{\prime} \stackrel{h^{\prime}}{\longrightarrow} C \stackrel{c h^{-}}{\longrightarrow} H N(A, I) ; c h^{\prime}$ is defined similarly using $c h_{\text {rht }}^{-}$. By Theorem 6.5.1, there is a homotopy between the two maps $C \rightarrow H N(A, I)$, and hence between the two maps $K(A, I)^{\prime} \rightarrow H N(A, I)$. Since this is a homotopy of functors from the small category of pairs $(A, I)$ to Simplicial Sets, it provides a natural homotopy between $c h$ and $c h^{\prime}$ as maps $K(A, I)^{\prime} \rightarrow H N(A, I)$.

\section{Acknowledgments}

We would like to thank Gregory Ginot and Jean-Louis Cathelineau for bringing the $c h_{*}=c h_{*}^{\prime}$ problem to our attention. We are also grateful to Christian Haesemeyer for several discussions about the relation between this paper and our joint paper [2].

\section{References}

1. J.-L. Cathelineau, $\lambda$-structures in Algebraic K-theory and Cyclic Homology, K-theory 4 (1991), 591606.

2. G. Cortiñas, C. Haesemeyer and C. Weibel, Infinitesimal cohomology and the Chern character to negative cyclic homology. Preprint, 2005, available at http://www.math.uiuc.edu/K-theory/0824.

3. G. Ginot, Formules explicites pour le charactère de Chern en $K$-théorie algébrique, Ann. Inst. Fourier $54,(2004)$ 2327-2355.

4. T. Goodwillie, Relative algebraic K-theory and cyclic homology, Annals Math. 124 (1986), 347-402.

5. M. Karoubi, Homologie cyclique et K-théorie. Astérisque 149, 1987.

6. C. Kassel, L'homologie cyclique des algèbres enveloppantes, Invent. Math. 91 (1988), 221-251.

7. J.-L. Loday, Cyclic homology, volume 301 of Grundlehren der Mathematischen Wissenschaften, Springer-Verlag, Berlin, 1992.

8. J.-L. Loday and D. Quillen, Cyclic homology and the Lie algebra homology of matrices, Commentarii Math. Helv. 59 (1984), 565-591.

9. C. Ogle and C. Weibel, Cyclic homology and relative $K$-theory, unpublished preprint, 1986.

10. D. Quillen, Rational Homotopy Theory, Annals Math. 90 (1969), 205-295.

11. M. Sweedler, Hopf algebras, Benjamin, 1969.

12. A. Suslin and M. Wodzicki, Excision in algebraic K-theory, Annals Math. 136 (1992) 51-122.

13. C. Weibel, An introduction to homological algebra. Cambridge Univ. Press, 1994. 